\title{
Analysis of Nursing Clinical Decision Support Requests and Strategic Plan in a Large Academic Health System
}

Kimberly Whalen ${ }^{1,5}$; Karen Bavuso2; Sharon Bouyer-Ferullo²; Denise Goldsmith³; Amanda Fairbanks²; Emily Gesner²; Charles Lagor2; Sarah Collins $2,3,4$

${ }^{1}$ Massachusetts General Hospital, Boston, MA; ${ }^{2}$ Partners Healthcare System, Wellesley, MA; ${ }^{3}$ Brigham and Women's Hospital, Boston, MA; ${ }^{4}$ Harvard Medical School, Boston, MA; ${ }^{5}$ University of Colorado Denver, Denver, CO

\section{Keywords}

Electronic health records, evidence-based nursing, hospital information systems, clinical decision support, nursing informatics

\section{Summary}

Objectives: To understand requests for nursing Clinical Decision Support (CDS) interventions at a large integrated health system undergoing vendor-based EHR implementation. In addition, to establish a process to guide both short-term implementation and long-term strategic goals to meet nursing CDS needs.

Materials and Methods: We conducted an environmental scan to understand current state of nursing CDS over three months. The environmental scan consisted of a literature review and an analysis of CDS requests received from across our health system. We identified existing high priority CDS and paper-based tools used in nursing practice at our health system that guide decision-making.

Results: A total of 46 nursing CDS requests were received. Fifty-six percent $(n=26)$ were specific to a clinical specialty; 22 percent $(n=10)$ were focused on facilitating clinical consults in the inpatient setting. "Risk Assessments/Risk Reduction/Promotion of Healthy Habits" $(n=23)$ was the most requested High Priority Category received for nursing CDS. A continuum of types of nursing CDS needs emerged using the Data-Information-Knowledge-Wisdom Conceptual Framework: 1) facilitating data capture, 2) meeting information needs, 3) guiding knowledge-based decision making, and 4) exposing analytics for wisdom-based clinical interpretation by the nurse.

Conclusion: Identifying and prioritizing paper-based tools that can be modified into electronic CDS is a challenge. CDS strategy is an evolving process that relies on close collaboration and engagement with clinical sites for short-term implementation and should be incorporated into a long-term strategic plan that can be optimized and achieved overtime. The Data-Information-Knowledge-Wisdom Conceptual Framework in conjunction with the High Priority Categories established may be a useful tool to guide a strategic approach for meeting short-term nursing CDS needs and aligning with the organizational strategic plan.

\section{Correspondence to:}

Kimberly Whalen MSN, RN

Massachusetts General Hospital

Boston, MA 02114

Email: Kwhalen3@partners.org
Appl Clin Inform 2016; 7: 227-237

http://dx.doi.org/10.4338/ACl-2015-10-RA-0128

received: October 7, 2015

accepted: February 1, 2016

published: April 13, 2016

Citation: Whalen K, Bavuso K, Bouyer-Ferullo S, Goldsmith D, Fairbanks A, Gesner E, Lagor C, Collins S.

Analysis of nursing clinical decision support requests and strategic plan in a large academic health system. Appl Clin Inform 2016; 7: 227-237

http://dx.doi.org/10.4338/ACI-2015-10-RA-0128 


\section{Background}

Clinical Decision Support (CDS) provides clinicians with knowledge and patient-specific information, intelligently filtered or presented at appropriate times in the care continuum, to enhance health and health care [1]. CDS increases the quality of care, improves health outcomes, helps to avoid errors and adverse events, improves efficiency, reduces costs, and increases provider and patient satisfaction [2]. CDS has been integrated into electronic health records (EHRs) to enhance nursing decision-making and evidence-based practice, as well as to augment workflow. Common examples are alerts pertaining to lab values that affect medication administration and alerts that provide evidence-based nursing interventions to the operating room nurse.

Partners Healthcare System (PHS)has a strong history of providing robust CDS interventions to clinicians. PHS has developed enterprise-wide knowledge management processes for specifying, developing, and maintaining CDS interventions. These knowledge management processes, such as the "CDS Lifecycle," (see section 5.1) are used operationally to support the collaborative specification, authoring, and maintenance of CDS assets $[3,4]$. The processes are especially critical as Partners is undergoing a major transition to replace its existing electronic systems with an integrated vendorbased EHR; a large-scale effort known as Partners eCare (PeC).

Prior to PeC, a majority of enterprise-wide nursing documentation was paper-based, which limited the opportunity to provide enterprise-wide CDS to nurses. Bakken et al. highlights the importance of incorporating information tools such as CDS into the nurse's clinical workflow [5]. According to Sim et al. CDS has the promise of bridging the gap between evidence and practice by applying evidence-based recommendations at the point of care [6]. PeC provides an opportunity to enhance nursing decision-making and evidence-based practice through CDS interventions. As an organization it is important to identify nursing CDS needs and find the optimal way to incorporate nursing CDS for short-term implementation and establish a long-term strategic plan.

\section{Objectives}

The aims of this project were (1) to understand the types of enterprise-wide CDS requests that nurses submit and (2) to establish a process for guiding short-term implementation and identifying long-term strategic nursing CDS goals.

\section{Methods}

To understand the type of CDS requests at Partners we conducted an environmental scan, which consisted of a literature review and an analysis of nursing CDS requests received from across our health system.

A literature review using CINAHL, Pubmed and MEDLINE was conducted to understand the current state of CDS, with a particular focus on nursing. The literature search used the following terms (with synonyms and closely related words): "clinical decision support" combined with "nursing", "conceptual framework" and "theory". The searches were not limited by study design or language of publication. Further studies were identified by examining the reference lists of all included articles. The literature review was used to identify a conceptual framework to categorize the spectrum of nursing CDS needs. Our literature search confirmed Anderson and Wilson's finding that there has been little research on theoretical models to support the development of CDS in nursing [7].

We chose the conceptual Data-Information-Knowledge-Wisdom (DIKW) model to frame the continuum of nursing CDS needs ( Figure 1), because it elucidates clinical and informatics gaps in CDS requested by nurses. Graves and Corcoran provided the Data, Information, Knowledge and Wisdom Framework as the first widely accepted framework for Nursing Informatics [8]. The American Nurses Association (2008) adopted the framework in its definition of Nursing Informatics $[8,9]$. According to Matney et al. the DIKW Framework provides a foundational framework for Nursing Informatics that allows nurses to connect practice with theory [8]. 
As a second part of the environmental scan, we asked nursing contacts at different Partners sites to identify existing CDS and paper-based interventions used in nursing practice that guide decisionmaking. Email communications were sent to nursing leadership seeking to understand the current state of CDS at their site and invited sites to submit requests for CDS interventions. Nursing leadership was asked to distribute a CDS request form to nurses at their site. The request form was developed by the PeC Clinical Informatics team and approved by the Partners CDS Committee, which is comprised of more than 50 members from different clinical professions, specialties, and sites. The form captures the necessary elements of current and future CDS interventions. Listed below were the questions established by Partners CDS Committee to be filled out by the requestor [10]:

- What do you want the CDS to do?

- Who are the intended recipients of the CDS?

- Explain the rationale for making the request.

- How is this handled in your institution today?

- Do you know how this could be solved in the new system?

- Does the CDS help PHS meet a regulatory requirement?Provide supporting documentaion.

- Does it improve patient safety?

- Is it required for Meaningful Use?

- Does it have financial implications?

- Does it assist clinician workflow?

We analyzed all of the requests received within a three-month period and categorized them according to the clinical professions. We identified the clinical profession who entered the data that triggered the CDS intervention and the clinical professions who received the CDS intervention's recommendation or action. We extracted and analyzed the requests for CDS interventions that specifically targeted nursing. The requests were categorized using two complementary frameworks: 1) a framework of high priority CDS categories established by Partners CDS Committee, and 2) the Data-Information-Knowledge-Wisdom Conceptual Framework.

\subsection{High Priority CDS Categories}

Prior to receiving requests, the CDS Committee established high priority categories for all CDS interventions. In developing these criteria, the committee considered the organization's strategic aims as well as safety and quality measures such as: regulations, patient safety metrics, Meaningful Use measures, return on investment measures, and workflow impact. The guiding principles for the high priority categories were patient safety, end user acceptance, informatics best practice, resources, organizational goals, evidence-based practice, research and teaching [10]. The resulting list of high priority categories was as follows:

- Allergy/Adverse Drug Reaction Documentation

- Pregnancy and Breastfeeding Status

- Indwelling Device Management

- Risk Assessments/Risk Reduction/Promotion of Healthy Habits

- Anticoagulation Management

- Peri-Operative Antibiotics

- Blood Ordering

- Infectious Disease Precautions

- Venous Thromboembolism Prevention

- Total Parenteral Nutrition

- Immunizations

- Restraints

- Code Status

- Labs which Impact Medication Administration/Ordering

- Fluid Management in Neonates

- Abnormal Test Result Management

- Referral Management

- High priority Chronic Diseases

C) Schattauer $2016 \quad$ K. Whalen et al.: Analysis of Nursing Clinical Decision Support Requests and Strategic Plan in a Large Academic Health System 
- High cost/risk intervention

- Other

We prioritized nursing CDS according to this list of high priority categories.

\subsection{Data-Information-Knowledge-Wisdom Conceptual Framework}

We then used the Data-Information-Knowledge-Wisdom Conceptual Framework, applying the following codes to each CDS request we received for nursing:

1. Facilitating data capture (example: does patient smoke?)

2. Meeting information needs (example: lab values that affect medication administration)

3. Guiding knowledge-based decision making (example: risk assessment for peripheral nerve damage in operating room)

4. Exposing analytics for wisdom-based clinical interpretation by the nurse (example: sepsis bundle)

The coding was performed by the author, and was validated in consensus meetings with three other investigators (SC, KB \& SB).

\section{Results}

During a three month span we received 200 enterprise wide CDS requests. Out of the 200 requests, 46 were nursing specific. We disregarded the remaining 154 requests because they were not specific to nursing. The types of request received by clinical focus area are summarized in $>$ Table 1 . The counts of different CDS request types are congruent with our experience. Specialty specific/Unique requests made up over half of the requests $(n=26,56 \%)$. The second largest group of nursing CDS requests received were related to consults based on assessment data $(n=10,22 \%)$. The breakdown of the $22 \%$ of consult requests was as follows: $(n=5,11 \%)$ for skin care consults, $(n=3,7 \%)$ for social work consults and $(\mathrm{n}=2,4 \%)$ for nutrition consults. The remainder of consults requested consisted of $(n=3,7 \%)$ Sepsis alerts, $(n=3,7 \%)$ Suicide Screen Precautions, $(n=2,4 \%)$ Morse Fall Score and $(\mathrm{n}=2,4 \%)$ Medication/Micromedix. The reason for the low number of medication requests were attributed to the existence of an existing legacy electronic medication administration record.

\subsection{High Priority Categories}

The distribution of nursing CDS requests within the high priority categories are summarized in - Table 2. The nursing CDS requests could fall into more than one of the High Priority Categories. The results of the analysis showed Risk Assessments/Risk Reduction/Promotion of Healthy Habits were among the most frequently requested $(n=23)$, followed by requests for nursing CDS that were in the High cost/risk intervention category $(n=15)$. The core of nursing care is to provide safe care, educate and promote health. The analysis displays an accurate representation of nursing needs and the tools nurses are looking for to provide care. The results suggest that nurses are looking for patient information at the right time to provide evidence based care.

\subsection{CDS requests along the Data-Information-Knowledge-Wisdom Framework}

Distribution of the nursing CDS requests according to the DIKW is summarized in $>$ Table 3 . The majority of nursing CDS requests received were in the Information category $(n=26,56 \%)$. This category pertains to CDS that provide nurses with information about their patient at the right time. For example, CDS that send alerts pertaining to lab values that affect medication administration. The Information category was followed by the Knowledge category $(n=15,33 \%)$, which refers to any CDS that provides an alert according to the information the nurse enters into the system based on 
nursing knowledge. For example, CDS that promotes accurate documentation of pressure ulcer stage based on nursing knowledge and recommends an appropriate plan of care. The Wisdom and the Data categories had the fewest amount of requests: 3 Nursing CDS requests fell into the Wisdom category $(7 \%)$ CDS that are wisdom based are activated when specific patient information is entered by the nurse applying his or her knowledge. Two requests fell into the Data category (4\%), which refers to any CDS that captures data.

\subsection{Process to guide both short-term implementation and long-term strategic goals to meeting nursing CDS needs}

The results from the analysis identified a process to guide both short-term implementation and long-term strategic goals to meet nursing CDS needs. This process aligned with the CDS lifecycle will guide an organization in the short-term and long-term implementation of CDS. The process identified during the analysis was:

1. Identify requests

2. Categorize by short-term and long-term CDS according to high priority categories and DIKW framework

3. Implement short-term CDS

4. Define requirements and resources needed for implementation of long term CDS

\section{Discussion}

In this paper we investigated enterprise-wide nursing CDS requests. In addition, we identified the high priority categories and the Data-Information-Knowledge-Wisdom (DIKW) model as a useful framework to guide short-term implementation and long-term strategic goals to meet nursing CDS needs. Our results are relevant to managing CDS interventions.

\subsection{CDS Life Cycle and Nursing CDS Request Process}

At Partners, we have implemented a so-called "CDS lifecycle" which captures the phases from a CDS request, to its prioritization, its design, its implementation, its testing, and it's monitoring ( $\triangleright$ Figure 2) [10]. The CDS lifecycle guides the process for managing CDS interventions including: identification, prioritization, specification, development, testing, and monitoring $[10,11]$. As part of this lifecycle, a strategic plan, or roadmap, could help guide the identification and prioritization of CDS interventions as outlined by Osheroff et al. who provided a roadmap for national action on clinical decision support [1]. The roadmap identifies three pillars for optimizing CDS: 1) best knowledge available when needed, 2) high adoption and effective use, and 3) continuous improvement of knowledge and CDS methods [1]. The process identified in the analysis along the CDS lifecycle will provide a guide to manage CDS needs in an organization. Short term and long term CDS can and should be worked on simultaneously, to continuously improve and update CDS needs. Short term and long term CDS require different tasks, resources and timeframes. It has been shown that organizational engagement with the CDS lifecycle can be used to optimize CDS interventions and identify new areas of need [12].

CDS interventions within electronic health records are intended to provide the right information, to the right person at the right time to enable evidence-based care [2]. Nursing CDS are specifically designed to support nursing decision making. Prior to an implementation of an electronic health record (EHR), paper-based tools provide decision support to nurses in the clinical setting. Yet, we found that identifying paper-based interventions that may be ripe for CDS is a challenge. The CDS Committee developed a standardized request form that would capture the current state of CDS and to evaluate new CDS requests [13]. Interestingly, our team received a non-trivial number of emails from nursing leaders and staff asking for scope clarification and definition of CDS interventions. This finding highlights the need for a useful tool to prioritize CDS requests along the CDS lifecycle to optimize meeting clinical needs over time. 


\subsection{High Priority Categories}

The second step of the CDS Lifecycle is to prioritize [10]. Partners CDS Committee established high priority categories for all CDS interventions. In developing these criteria, the committee considered the organization's strategic aims as well as safety and quality measures. The guiding principles for the high priority categories were patient safety, end user acceptance, informatics best practice, resources, organizational goals, evidence-based practice, research and teaching [10]. The Centers for Medicare $\&$ Medicaid Services (CMS) requires eligible hospitals to use clinical decision support to improve performance on high-priority health conditions [11]. These clinical decision support interventions must be explicitly related to the CMS defined high-priority health conditions [11]. The high priority categories established by Partners CDS Committee is a useful tool in the second step of the CDS Lifecycle [10]. It establishes what nursing CDS interventions should be brought to the next state in the lifecycle.

\subsection{Conceptual Framework for Nursing CDS Requests}

The DIKW framework could be a useful tool to enhance the request and prioritization phases of the CDS lifecycle. It establishes and prioritizes the informational needs that CDS interventions are currently providing to nurses and what nurses will expect from CDS long term. We propose using the DIKW framework in conjunction with the High Priority Categories to drive long term strategic planning and help in prioritizing nursing CDS interventions at the beginning of the CDS Lifecycle.

Our use of the DIKW framework identified a continuum of nursing CDS needs: 1) facilitating data capture, 2) meeting information needs, 3) guiding knowledge-based decision making, and 4) exposing analytics for wisdom-based clinical interpretation by the nurse. These results are confirmed in a recent expert survey of the most highly ranked research topics [14] for nursing informatics which includes: 1) the development of systems that provide real-time feedback to nurses to improve safety, 2) the impact of systems on nursing care, 3) decision support systems for nurses, and 4) the impact of systems on workflow [14]. It was also noted that design research and methods to evaluate systems impacting nursing workflow has been limited [14]. As organizations advance the types of CDS interventions provided to nurses the DIKW framework can provide a roadmap to identify and evaluate CDS interventions intended to achieve the topics outlined by Dowding et al [14].

The first level in the DIKW framework pertains to nursing CDS that facilitate data capture. Matney et al. explains data has little or no meaning in isolation [8]. In the nursing assessment, the nurse documents the answers to the question, "Does the patient smoke? "The nurse documents a yes/ no value. At this level the nurse just enters data to a question. There is no nursing judgment or knowledge needed to document this data.

The second level in the framework is meeting information needs. In the analysis, CDS that meet information needs were the most frequently requested. This CDS provides information to the nurse about their patient at the right time. CDS that meet information needs are alerts that provide the nurse with relevant information pertaining to a specific patient. Matney et al. expands on Graves and Corcoran framework explaining information may be thought of as "data plus meaning" [8]. Common examples of information CDS requests were for alerts pertaining to lab values that affect medication administration.

The third levels in the framework are CDS that guide knowledge-based decision making. Matney et al. suggests knowledge is derived by discovering patterns and relationships between types of information [8]. The knowledge based CDS category refers to any CDS that provides an alert according to the information the nurse enters into the system based on nursing knowledge. An example is a CDS that recommends evidence-based nursing interventions to the operating room nurse. The CDS intervention is activated when a nurse applies his or her knowledge by documenting that a patient has two or more risk factors for peripheral nerve injury. The research by Bouyer-Ferullo et al. on peripheral nerve injury showed implementation of CDS supported the operating room nurses with patient assessment and diagnosing for peripheral nerve injury risk factors and offered evidence-based nursing interventions [15]. Nursing decisions can be supported by technology when caring for patients who are at higher risk of injury [15]. 
The final level in the DIKW framework is CDS that expose analytics for wisdom based clinical interpretation. Matney et al state the difference between knowledge and wisdom is like the difference between memorizing and understanding-there is a process of internalization [8]. CDS that are wisdom based are activated when specific patient information is entered by the nurse applying his or her knowledge. The nurse leverages his or her wisdom when deciding if the evidence-based recommendations provided by the CDS intervention are appropriate for that patient. The sepsis bundle is an example of a wisdom based CDS. The nurse enters patient specific information, such as temperature and blood pressure. Based on entered patient data, the CDS intervention sends an alert notifying the nurse the patient is at risk for sepsis. The nurse would use his or her experience and knowledge-based wisdom to determine if the recommendation is accurate and relevant for this specific patient. Benner summarizes that nursing wisdom is based on clinical judgment and a thinking inaction approach that encompasses intuition, emotions, and the senses [16].

CDS categorized as wisdom may be valuable in improving patient safety. The alert for a novice nurse may correlate all the data which indicates sepsis that his/her knowledge base would not be able to link together. The experienced nurse may already realize by the time the alert is activated that the patient is at risk for sepsis. The experienced nurse will evaluate the available data and along with her experience, intuition and senses know that the patient is at risk. Applying Benner et al. "Noviceto Expert Nursing Practice framework" Courtney et al. concluded that CDS should be tailored to the differing needs of nurses at various clinical levels [17]. There has been much attention focused on the best sensitivity and specificity for CDS sepsis risk alerting. Perhaps tailored interventions based on clinical levels of expertise could be used to stratify alert settings.

Our analysis showed that the majority of CDS nursing requests were for 'information' and 'knowledge. This could be due to the fact that many 'data' CDS requests existed in current systems and those needs were met. CDS that fall into the information and knowledge categories may be the most easily understood and obtainable by staff at this juncture. Opportunities for CDS that fall into the wisdom category may require more experience and familiarity with advanced capabilities of CDS and real-time EHR analytics. Based on our findings, our team has identified the goal to obtain a future state of active CDS interventions within the wisdom category.

\section{Limitations}

The scope of this project was limited to the identification of CDS needs as an initial step in the CDS lifecycle. The implementation of identified needs, which is resource-dependent and must be prioritized among other competing project needs, was out of scope of this project.

This project was limited to a 3-month time span for obtaining requests. Email communications were sent to nurses at PHS sites asking for the submission of nursing CDS requests; however, there may have been nurses at PHS that did not receive these email communications but do have CDS requests. Additionally, PeC system is currently being configured and is not yet implemented for clinical use, we anticipate there may be a greater and different response from nurses after "go-live". Finally, we coached site contacts in how to describe and define CDS to nurses at their sites, but this coaching may benefit from a more formal approach to ensure nurses understand what CDS is and can interpret their needs appropriately to submit requests.

\section{Conclusion}

Identification of CDS needs is an initial step in the CDS lifecycle. Osheroff et al. highlights the importance of the appropriate use of CDS to optimize patient safety, care quality, patient centeredness, and cost effectiveness [1]. This requires systems to provide evidence based knowledge [1]. In our institution, the strategic process for nursing CDS is evolving and relies on close collaboration and engagement with clinical sites. To effectively distribute limited resources in meeting the highest priority needs, the Data-Information-Knowledge-Wisdom Conceptual Framework along with the High Priority Categories may be useful tools to incorporate CDS during short-term implementation and a long-term strategic planning. CDS that meet Data needs are likely to be frequent in short term im- 
plementations, whereas CDS that meet Wisdom needs are likely candidates for long-term strategic planning.

\section{Clinical Relevance}

The Data-Information-Knowledge-Wisdom Conceptual Framework in conjunction with High Priority Categories may be a useful mechanism to guide a strategic approach for meeting nursing CDS needs and aligning with the organizational strategic plan. There are many concerns nursing leadership need to take into account when implementing a system, such as alert fatigue. The DIKW Framework may be a useful tool to prioritize CDS along the CDS lifecycle.

\section{Conflict of interest}

None of the listed authors have any financial or personal relationships with other people or organizations that may inappropriately influence of bias the objectivity of submitted content and /or its acceptance of publication in this journal.

\section{Human Subjects Protections}

Institutional Review Board (IRB) approval was not required, no human subjects were involved in the research.

\section{Acknowledgments}

The authors thank Diana Skiba, PhD for her academic advising. We acknowledge Partners CDS Committee, other members of thePeC team, and all nurses at Partners that contributed to this project. 


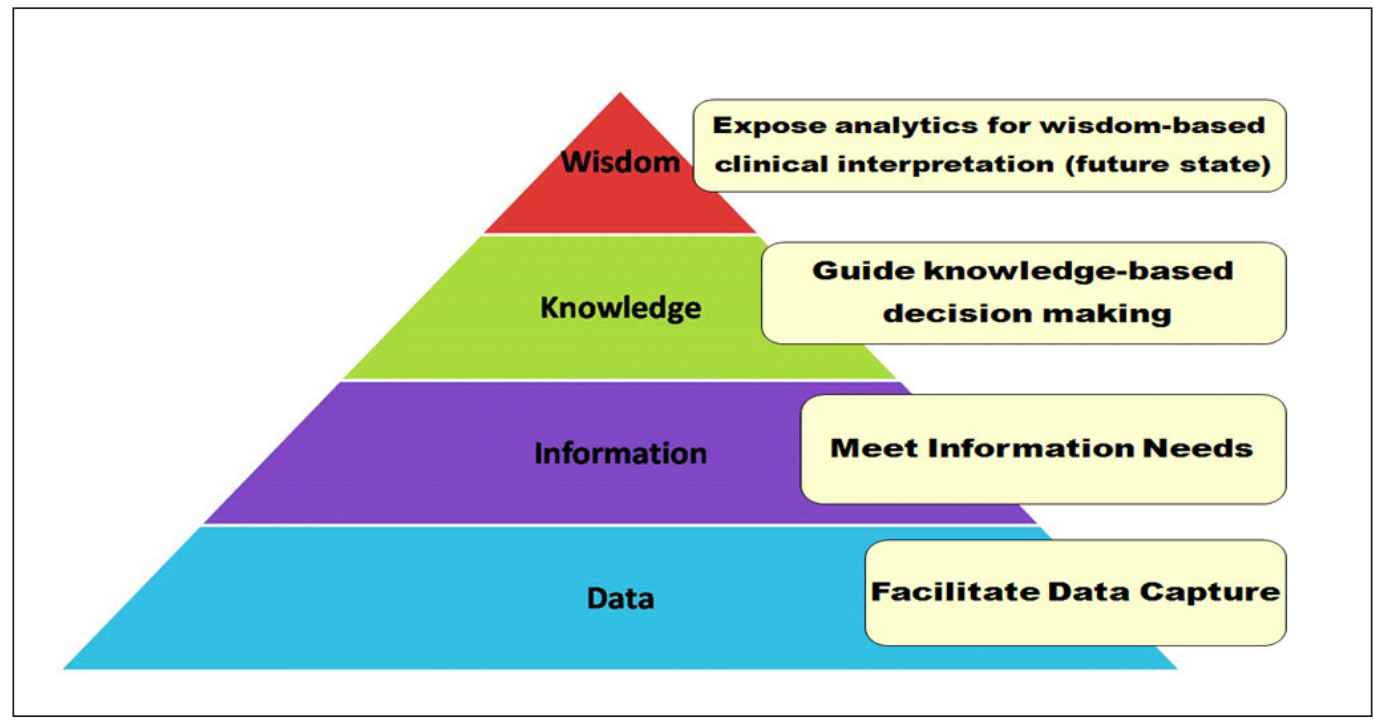

Fig. 1 Nursing CDS needs along the Data-Information-Knowledge-Wisdom (DIKW) Framework

Fig. 1 Clinical Decision Support (CDS) LifeCycle

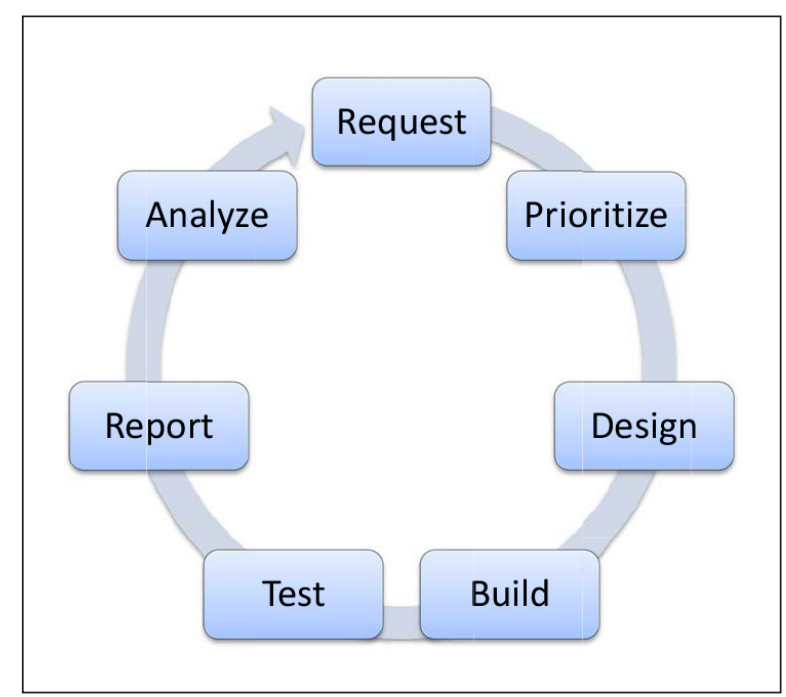


Table 1 Types of Nursing Requests

\begin{tabular}{|l|r|}
\hline \multicolumn{2}{|l|}{ Nursing CDS Requests Received by Clinical Focus Area } \\
\hline Nursing CDS Requests & Request Rate \% (n/total) \\
\hline Specialty specific/Unique & $56 \%(26 / 46)$ \\
\hline Consults based on assessment data & $22 \%(10 / 46)$ \\
- Skin care consults & $11 \%(5 / 46)$ \\
- Social work consults & $7 \%(3 / 46)$ \\
- Nutrition consults & $4 \%(2 / 46)$ \\
\hline Sepsis alerts & $7 \%(3 / 46)$ \\
\hline Suicide screen precautions & $7 \%(3 / 46)$ \\
\hline Morse fall score & $4 \%(2 / 46)$ \\
\hline Medication/Micromedix & $4 \%(2 / 46)$ \\
\hline Total & $100 \%$
\end{tabular}

Table 2 High Priority Categories of Nursing Requests

\begin{tabular}{|l|c|}
\hline High Priority Categories of Nursing CDS Requests Received & \\
\hline High Priority Categories & \# of Requests (n) \\
\hline Risk Assessments/Risk Reduction/Promotion of Healthy Habits & 23 \\
\hline High cost/risk intervention & 15 \\
\hline Anticoagulation Management & 12 \\
\hline Labs which impact medication administration /ordering & 11 \\
\hline Abnormal test results & 11 \\
\hline Management Referral & 8 \\
\hline Other & 3 \\
\hline Venous thrombembolism prevention & 2 \\
\hline Indwelling device management & 2 \\
\hline Pregnancy and breastfeeding status & 1 \\
Code Status & 1 \\
\hline Restraints & 1 \\
\hline Infectious disease precautions & 1 \\
\hline
\end{tabular}

Table 3 Analysis of CDS Request along the Data-Information-Knowledge-Wisdom Framework

\begin{tabular}{|l|l|l|}
\hline \multicolumn{2}{|l|}{ Data Information Knowledge Wisdom Categorization Results for Nursing CDS Requests } \\
\hline Framework Concepts & Request Rates (n/total) & Examples \\
\hline Data & $4 \%(2 / 46)$ & Medication information/Micromedix \\
\hline Information & $56 \%(26 / 46)$ & $\begin{array}{l}\text { Alerts pertaining to lab values that affect medication admin- } \\
\text { istration }\end{array}$ \\
\hline Knowledge & $33 \%(15 / 46)$ & $\begin{array}{l}\text { CDS that recommends evidence-based nursing interventions } \\
\text { to the operating room nurse. }\end{array}$ \\
\hline Wisdom & $7 \%(3 / 46)$ & Sepsis alert \\
\hline Total & $100 \%(46 / 46)$ & \\
\hline
\end{tabular}




\section{References}

1. Osheroff JA, Teich JM, Middleton B, Steen EB, Wright A, Detmer DE. A roadmap for national action on clinical decision support. J Am Med Inf Assoc 2007; 14(2): 141-145. doi:10.1197/jamia.M2334

2. Centers for Mediacare \& Medicaid Services (CMS).US Deparment of Health and Human Services. EHR Incentive Programs, Clinical Decision Support. http://www.cms.gov/Regulations-and Guidance/Legislation/EHRIncentivePrograms/Downloads/ClinicalDecisionSupport_Tipsheet-.pdf. Accessed May, 9 2015.

3. Kawamoto K, Lobach D, Willard H, Ginsburg G. A national clinical decision support infrastructure to enable the widespread and consistent practice of genomic and personalized medicine. BMC Med Inform Decis Mak 2009; 9: 17. doi: 10.1186/1472-6947-9-17

4. Hongsermeier T, Kashyap V, Sordo M. In: Clinical Decision Support: the Road Ahead. Greenes RA, editor. Boston: Elsevier Academic Press; 2007. Knowledge management infrastructure: evaluation at Partners HealthCare System; pp. 447-467.

5. Bakken S, Currie LM, Lee N-J, Roberts WD, Collins S, Cimino JJ. Integrating evidence into clinical information systems for nursing decision support. Int J Med Inf 2008; 77(6): 413-420. doi:10.1016/j.ijmedinf.2007.08.006

6. Sim I, Gorman P, Greenes RA, Haynes RB, Kaplan B, Lehmann H, Tang PC. Clinical decision support systems for the practice of evidence-based medicine. J Am Med Inform Assoc 2001; 8 (6): 527-534.

7. Anderson J, Wilson P. Clinical decision support systems in nursing synthesis of the science for evidencebased practice. Computers, Informatics, Nursing 2008; 26(3): 151-158.

8. Matney S, Brewster PJ, Sward KA, Cloyes KG, Staggers N. Philosophical approaches to the nursing informatics data-information-knowledge-wisdom framework. Adv Nurs Sci 2011; 34(1): 6-18. doi:10.1097/ANS.0b013e3182071813

9. Nursing Informatics. Scope and Standards of Practice. Silver Spring, MD: American Nurses Association (ANA) 2008; 1: 177.

10.Lagor C. The Clinical Decision Support Life-Cycle. Retrieved from http://s3.amazonaws.com/rdcmshimss/files/production/public/2015Conference/handouts/96_1428607926574_4.pdf

11. Centers for Mediacare \& Medicaid Services (CMS). US Deparment of Health and Human Services. http://www.cms.gov/Regulations-and-Guidance/Legislation/EHRIncentivePrograms/downloads/ Stage2_EPCore_6_ClinicalDecisionSupport.pdf Accessed May, 92015.

12. Maviglia S, Sordo M. Practical approaches to knowledge management: Focus on clinical decision support. MEDINFO 2015. Proceedings of the 15th World Congress on Health (Medical) Informatics; 2015 Aug 19-23, Sao Paulo, Brazil. http://medinfo2015.com/wpcontent/uploads/2015/06/Practical-Approaches-toKnowledge-Management-Focus-on-Clinical-Decision-Support.pdf

13.Xiang S, Bouyer-Ferullo S, Lagor C, Maviglia S, Yoshida E. The evolution of a clinical decision support request form. American Medical Informatics Association, 2015.

14. Dowding D, et al. International priorities for research in nursing informatics for patient care. Stud Health Technol Inform 2013; 192: 372-376.

15. Bouyer-Ferullo S, Androwich I, Dykes P. Clinical decision support and perioperative peripheral nerve injury: A quality improvement project. CIN: Computers, Informatics, Nursing 2015; 33(6): 238-248. doi:10.1097/CIN.00000000000148

16. Benner P. The wisdom of our practice. The American Journal of Nursing2000; 100(10): 99-105.

17. Courtney K, Alexander G, Demiris G. Information technology from novice to expert: implementation implications. Journal of Nursing Management 2008; 16: 692-699. 